\title{
POLYDISPERSITY INDEX FROM LINEAR VISCOELASTIC DATA: UNIMODAL AND BIMODAL LINEAR POLYMER MELTS.
}

\author{
J. Llorens ${ }^{1}$, E. Rudé ${ }^{1}$ and R. M. Marcos ${ }^{2}$
}

Correspondence to: J. Llorens (E-mail: Ilorens@angel.qui.ub.es)

${ }^{1}$ Chemical Engineering Department, University of Barcelona, C/ Martí i Franquès, 1, 08028 Barcelona, Spain

${ }^{2}$ Mechanical Engineering Department, ETSE, Rovira i Virgili University, Avda. Països Catalans, 26, 43007 Tarragona, Spain

\section{Abstract}

This article describes a method for determining the polydispersity index $I_{\mathrm{p} 2}=$ $\overline{\mathrm{M}}_{z} / \overline{\mathrm{M}}_{\mathrm{w}}$ of the molecular weight distribution (MWD) of linear polymeric materials from linear viscoelastic data. The method uses the Mellin transform of the relaxation modulus of a simple molecular rheological model. One of the main features of this technique is that it enables interesting MWD information to be obtained directly from dynamic shear experiments. It is not necessary to achieve the relaxation spectrum, so the ill-posed problem is avoided. Furthermore, a determinate shape of the continuous MWD does not have to be assumed in order to obtain the polydispersity index. The technique has been developed to deal with entangled linear polymers, whatever the form of the MWD. The rheological information required to obtain the polydispersity index is the storage $G^{\prime}(\omega)$ and loss G" $(\omega)$ moduli, extending from the terminal zone to the plateau region. The method provides a good agreement between the proposed theoretical approach and the experimental polydispersity indices of several linear polymers for a wide 
range of average molecular weights and polydispersity indices. It is also applicable to binary blends.

Keywords: polymer rheology, polydispersity index, molecular weight distribution.

\section{Introduction}

Most of the properties of a polymeric material are related to its molecular weight distribution (MWD). The conventional analytical methods for determining the MWD require dissolution of the sample. However, some polymers are either difficult to dissolve or insoluble in common solvents. Furthermore, if available equipment is not fitted with a sensor for determining absolute molecular weight, standard polymers are needed to calibrate the instrument. In contrast, some rheological techniques do not come up against these problems. The rheological technique would appear to be a powerful tool for the molecular characterization of polymeric materials. Indeed, in recent years much research has aimed to define the interrelationship between polymer rheological information and molecular weight distribution [1-10].

In many technical applications, it is not necessary to know the whole molecular weight distribution in order to characterize the material from a molecular point of view; knowing an average molecular weight and a polydispersity index is sufficient. The parameters normally used are the average molecular weight $\overline{\mathrm{M}}_{\mathrm{w}}$ and a polydispersity index $\overline{\mathrm{M}}_{\mathrm{w}} / \overline{\mathrm{M}}_{\mathrm{n}}$ or $\overline{\mathrm{M}}_{z} / \overline{\mathrm{M}}_{\mathrm{w}}$. 
It is widely accepted that the rheological parameters directly related to $\bar{M}_{w}$ and the polydispersity of the MWD, $\overline{\mathrm{M}}_{z} / \overline{\mathrm{M}}_{\mathrm{w}}$, are the zero shear rate viscosity, $\eta_{0}$, and the steady-state compliance, $\mathrm{J}_{\mathrm{e}}^{0}$, respectively.

The zero shear rate viscosity is a characteristic parameter for the entanglement density between chains within a material, and hence it is very susceptible to chain length. The steady state compliance is a parameter for melt elasticity which characterizes the chain elastic deformation capacity between entanglements with regard to their static equilibrium position. It is also a measure of the elastic energy that is accumulated during flow and recoverable when the stress is removed. Therefore, it is very susceptible to the high molecular weight fractions of the material $[11,12]$.

The dependence of $\eta_{0}$ on the weight average molecular weight can be described by the following power law:

$$
\eta_{o}=k_{E} \cdot \bar{M}_{w}^{\alpha}
$$

where $\mathrm{k}_{\mathrm{E}}$ is the constant of proportionality and $\alpha$ generally lies between 3.3 and 3.7, except for extremely broad blends, for which higher values are observed [13-16]. $\alpha$ is often taken as 3.4 , since this is the value usually obtained for most linear polymers investigated.

The dependence of steady state compliance on molecular weight distribution has also been widely studied because of its importance in polymer processing. Mieras and van Rijn [17] observed that in the case of polydisperse entangled polymer melts (polystyrene, PS, and polypropylene, PP) $\mathrm{J}_{\mathrm{e}}^{0}$ is not dependent on molecular weight but is strongly influenced by the width of the MWD, characterized by the parameter $\overline{\mathrm{M}}_{z} / \overline{\mathrm{M}}_{\mathrm{w}}$. This finding was consistent with similar 
measurements in polydimethylsiloxane (PDMS) and PS melts, with $1<$ $\overline{\mathrm{M}}_{\mathrm{z}} / \overline{\mathrm{M}}_{\mathrm{w}}<3$, taken by Mills [18], who proposed the following empirical relationship:

$$
J_{e}^{0} \propto\left(\frac{\bar{M}_{z}}{\bar{M}_{w}}\right)^{3.7}
$$

which has since been widely accepted.

Later, another empirical correlation was proposed by Agarwal [19] to relate $\mathrm{J}_{\mathrm{e}}^{0}$ and molecular weight averages

$$
J_{e}^{0} \propto \frac{\bar{M}_{z} \bar{M}_{z+1}}{\bar{M}_{n} \bar{M}_{w}}
$$

this being derived by testing data from a set of binary blends of polystyrene reported in the literature. For the particular case of a logarithmic-normal MWD, Eq. (3) can be rewritten as

$$
J_{e}^{0} \propto\left(\frac{\bar{M}_{z}}{\bar{M}_{w}}\right)^{4}
$$

The two equations (2) and (4) give practically the same prediction not only for the case of binary blends but also for the continuous broad distribution [20].

In addition, several blending laws have also been proposed for estimating the effect of polydispersity on the linear viscoelastic properties of polymers. The simplest one is Ninomiya's linear blending law [21] for a mixture of two monodisperse polymers with molecular weights $M_{1}$ and $M_{2}$ :

$$
H \leftarrow=w_{1} H_{1} \leftarrow / \lambda_{1}{ }^{-}+w_{2} H_{2} \leftarrow / \lambda_{2}^{-}
$$

where $H(\tau)$ is the relaxation spectrum of the mixture, $H_{1}(\tau)$ and $H_{2}(\tau)$ are those of the individual pure components, and $w_{1}$ and $w_{2}$ are their weight fractions. The quantity $\lambda_{\mathrm{i}}$ is a dimensionless shift factor that modifies the relaxation times $\tau$ of 
molecules of component $i$ relative to their values in the pure state. These shift factors account for the effect of the molecular surroundings on the relaxation time of each molecule. The presence of shorter chains speeds up the relaxation time of longer ones, and vice versa. A simple shift factor that does this is $\lambda_{i}=M / M_{i}{ }^{\gamma}$, where $\bar{M}$ is an average molecular weight of the MWD and $\delta$ is a constant.

Assuming that the viscosity $\eta_{i}$ and the steady-state compliance $J_{e i}$ of the pure components are related to the molecular weight $\mathrm{M}_{\mathrm{i}}$ as [20]:

$$
\begin{gathered}
\eta_{i}=\int_{0}^{\infty} H_{i} \lessdot g \tau \propto M_{i}{ }^{3} \\
J_{e i} \cdot \eta_{i}^{2}=\frac{1}{\eta_{i}{ }^{2}} \int_{0}^{\infty} \tau H_{i} \lessdot d \tau \propto M_{i}{ }^{6}
\end{gathered}
$$

this simple blending law predicts that $\mathrm{J}_{\mathrm{e}}^{0}$ is proportional to $\bar{M}_{z} / \bar{M}_{w}$ if $\lambda_{\mathrm{i}}$ is assumed to be $\bar{M}_{w} / M_{i}{ }^{\lambda}$ and proportional to $\bar{M}_{z+1} \bar{M}_{z+2} Z \bar{M}_{w} \bar{M}_{z}$ if $\lambda_{\mathrm{i}}$ is assumed to be $\bar{M}_{w} / M_{i}$. Kurata [20] proposed a variation of equation (5) based on a mole-basis additivity instead of the weight-basis:

$$
H \leftarrow=x_{1} H_{1} \leftarrow / \lambda_{1}+x_{2} H_{2} \leftarrow / \lambda_{2}-
$$

where $x_{i}$ represents the mole fraction of component $i$ in the blend. If this is combined with $\lambda_{i}=\bar{M}_{w} / M_{i}$, it leads to the Agarwal equation of $\mathrm{J}_{\mathrm{e}}^{\circ}$ (Eq. 3).

Higher-order blending laws have also been proposed, for example, the quadratic law of Bogue et al. [22]:

$$
H \lessdot w_{1}^{2} H_{1} \ll / \lambda_{1} \ni 2 w_{1} w_{2} H_{12} \ll / \lambda_{12} \ni w_{2}^{2} H_{2} \ll / \lambda_{2}^{-}
$$

and the cubic power-law of Kurata [23]. These quadratic and cubic blending laws, with the empirical proportionality of $\eta_{0}$ to $\bar{M}_{w}{ }^{3.4}$ and certain assumptions 
about the cross terms, predict proportionality to $\bar{M}_{z} / \bar{M}_{w}{ }^{2}$, and $\bar{M}_{z} / \bar{M}_{w}$, respectively [20, 23].

There are two theoretical approaches to predicting the dependence of $J_{\mathrm{e}}^{0}$ on polydispersity in the literature. Graessley [12] estimated the effect of polydispersity on $\mathrm{J}_{\mathrm{e}}^{0}$ by working out the equations given by the molecular theory of viscoelasticity developed by Doi and Edwards for the dynamics of entangled linear polymeric liquids $[24,25]$. This molecular theory is based on the idea of a tube model to represent the mutual constraints on configurational rearrangement of the chains. According to this theory, Graessley [12] found the following $\mathrm{J}_{\mathrm{e}}^{0}$ dependence on average molecular weights:

$$
J_{e}^{0} \propto \frac{\bar{M}_{z+2} \bar{M}_{z+3} \bar{M}_{z+4}}{\bar{M}_{w} \bar{M}_{z} \bar{M}_{z+1}}
$$

Similarly, Bird et al. [26], using the kinetic theory of polymeric liquids, or phase-space theory, found the following relationship between steady-state compliance and average molecular weights

$$
J_{e}^{0} \propto \frac{\bar{M}_{z+1} \bar{M}_{z+2}}{\bar{M}_{w} \bar{M}_{z}}
$$

Equations (10) and (11) show that both theoretical approaches predict that the elastic behaviour of polymer melts, characterized by $\mathrm{J}_{\mathrm{e}}^{0}$, is independent of molecular weight for a monodisperse system but is strongly dependent on the MWD for a polydisperse one, which is qualitatively in accordance with experimental results. However, despite $\mathrm{J}_{\mathrm{e}}^{0}$ being quite sensitive to the presence of high molecular weight components, the Doi-Edwards prediction is almost certainly too strong. If one assumes a logarithmic-normal type distribution, the Doi-Edwards theory predicts that: 


$$
J_{e}^{0} \propto\left(\frac{\bar{M}_{z}}{\bar{M}_{w}}\right)^{9}
$$

which reveals a marked discrepancy with the experimental results. In contrast, the phase-space theory predicts a fourth power scaling relationship (Eq. 4), which is in close agreement with experimental findings (Eq. 2).

It should be pointed out that the constant of proportionality is, in all cases, the reciprocal of the plateau modulus, $\mathrm{G}_{\mathrm{N}}^{0}$. Similarly, all the above relationships emphasize the importance of the higher molecular weight averages in determining the steady-state compliance.

Other determinations of the polydispersity index from rheological measurements have been successfully used [27-30]. All of them, however, are based on phenomenological hypotheses or empirical rules and some are only valid for a particular class of polymer. Moreover, it is necessary to assume a type of distribution law (Weslau (logarithmic-normal), Schulz, Poisson or Flory type) for a quantitative analysis, as otherwise only qualitative information is available.

The present study describes a quantitative rheological method for calculating the polydispersity index, one that is based on a molecular theory and which can be applied to any kind of polymer sample, regardless of the form of the distribution. The rheological information required is the master curves of the storage (G'- $\omega)$ and loss $\left(G^{\prime \prime}-\omega\right)$ moduli, extending from the terminal to the plateau zones.

\section{Theory}


Most of the molecular models used to infer the molecular weight of polymer samples from linear viscoelastic data are based on the tube and reptation concept of de Gennes [25]. However, it has proved difficult to incorporate polydispersity into these models as the effect of the polydisperse environment on the relaxation times of the individual chains has to be taken into account. Graessley [31] introduced this polydispersity effect by postulating that the terminal relaxation time $\tau_{i}$ depends on two non-correlated processes: (i) the reptation of the chain and (ii) the constraint release due to the motion of the surroundings, which results in the tube renewal effect, a concept originally developed by both Klein [32] and Daoud and de Gennes [33].

One of the most successful approaches to predicting the MWD of polydisperse polymer samples is the double reptation mixing rule, independently derived by both Tsenoglou [34] and des Cloizeaux [35] from the entanglement concept and the double reptation mechanism, respectively. Recently, other authors $[5,7]$ have refined the double reptation theory by simultaneously taking into account tube renewal and the double reptation mechanism of the chains.

The molecular model used in the present study was formulated and analysed in a recent article [8]. Like most of the molecular models described in the literature, this model is applicable to polymeric systems when all the chains are long enough to form an entangled polymer network.

The model establishes that the relaxation function is well-approximated as a simple exponential function, in reasonable agreement with the well-known tube model for chain reptation in monodisperse polymers [24]. However, the experimental spectrum for monodisperse polymers is broader than predicted by 
the tube model. On the other hand, it is assumed that the effects of constraint release on the relaxation time, $\tau_{i}$, of a chain of molecular weight $M_{i}$, depend on an average molecular weight, $\bar{M}$, that sets the effect of the environment where the molecules reptate, according to

$$
\tau_{\mathrm{i}}=\left(\mathrm{k}_{\mathrm{E}} / G_{N}^{o}\right) \cdot \bar{M}^{\alpha-\beta} \cdot \mathrm{M}_{\mathrm{i}}^{\beta}
$$

In this equation the parameters $\mathrm{k}_{\mathrm{E}}$ and $\alpha$ are the same as previously described for Eq. (1), which relates $\eta_{0}$ and $\bar{M}_{w}, G_{N}^{o}$ is the plateau modulus and $\beta$ is a parameter which determines the contribution of the molecular weight of a chain in its relaxation time. Given a polymer with a definite MWD, Eq. (13a) can be rewritten as

$$
\tau_{\mathrm{i}}=\mathrm{K}^{\prime} \mathrm{M}_{\mathrm{i}}^{\beta}
$$

Other models assume that the relaxation function of probe chains can be expressed as a product of "chain reptation" and "constraint release" relaxation processes [31].

It is known that probe chains dispersed in a higher molecular weight matrix have their relaxation slowed, consistent with Eq. (13a). However, there is a discrepancy between the proposed model predicting that suppression of constraint release does not broaden the relaxation function and experiments, which show broadening $[36,37]$.

From a molecular point of view, polymers are characterized by their MWD, $\mathrm{W}(\mathrm{M})$, whereas the material function of linear viscoelasticity is often taken to be the relaxation modulus, $\mathrm{G}(\mathrm{t})$. In this model the normalized linear relaxation modulus for a polydisperse polymer is formulated by the following expression

$$
G(t)=G_{N}^{o} \int_{0}^{\infty} \exp \left(\frac{-t}{\tau_{i}}\right) W(M) d M
$$


Here we expand the previous molecular model [8] to derive a polydispersity index, for any kind of molecular weight distributions, from linear viscoelastic data. The study uses the integral transforms of the relaxation modulus, derived from classical viscoelastic theory, and the proposed molecular model. The expression for a polydispersity index is achieved as follow:

From classical viscoelastic theory [38] the relaxation modulus is related to the dynamic moduli by the following relationships:

$$
\begin{aligned}
& G(t)=\frac{2}{\pi} \int_{0}^{\infty} \frac{G^{\prime}}{\omega} \sin t g t \omega \\
& G(t)=\frac{2}{\pi} \int_{0}^{\infty} \frac{G^{\prime \prime}}{\omega} \cos \omega t g \omega
\end{aligned}
$$

In order to connect aspects of the molecular weight distribution with the dynamic moduli we combine Eqs. (14) and (15) through their Mellin transforms. The Mellin transform $f_{M}(s)$ of an arbitrary function $f(t)$ is defined by

$$
f_{M}(s) \equiv \int_{0}^{\infty} t^{s-1} f<d t
$$

where the parameter $s$ is the transform variable. If $f(t)=e^{-t / \lambda}$, the Mellin transform is, for $s>0$ :

$$
f_{M}(s) \equiv \int_{0}^{\infty} t^{s-1} e^{-t / \lambda} d t=\lambda^{s} \cdot \Gamma
$$

$\Gamma(\mathrm{s})$ being the gamma function of parameter $s$. Interesting properties of the Mellin transform can be used to solve problems of classical physics [39]. In particular, the Mellin transform is usefull to calculate moments of time functions [40]. Thus, the Mellin transform of the relaxation modulus expressed in terms of the relaxation spectrum $\left(G(t)=\int_{-\infty}^{\infty} H(\lambda) \cdot e^{-t / \lambda} \cdot d \ln \lambda\right)$ gives:

$$
G_{M}(s)=\int_{-\infty}^{\infty} H(\lambda) \int_{0}^{\infty} t^{s-1} \cdot e^{-t / \lambda} \cdot d t \cdot d \ln \lambda=\Gamma(s) \int_{-\infty}^{+\infty} H(\lambda) \cdot \lambda^{s} \cdot d \ln \lambda
$$


where $\int_{-\infty}^{+\infty} H(\lambda) \cdot \lambda^{s} \cdot d \ln \lambda$ is the sth moment of the of the relaxation spectrum. Moreover, polydispersity indexes of the relaxation spectrum can be obtained by the quotients of these moments. Therefore, if we know the relation between the relaxation times and the polymer molecular weights, Eq. (13), we can derive molecular polydispersity indexes. Then, taking into account Eq. (13b), the Mellin transform of the relaxation modulus (Eq. 14), $\mathrm{G}_{\mathrm{M}}(\mathrm{s})$, when $s>0$ is:

$G_{M}(s) \equiv G_{N}^{o} \int_{0}^{\infty}\left(\int_{0}^{\infty} t^{s-1} \exp \left(\frac{-t}{K^{\prime} M^{\beta}}\right) d t\right) W(M) d M=G_{N}^{o} \Gamma-K^{\prime s}-\int_{0}^{\infty} M^{\beta s} W M d M$

where $\int_{0}^{\infty} M^{\beta s} W \mathbb{d} M$ is the $\beta$ sth moment of the molecular weight distribution, $\left\langle\mathrm{M}^{\beta s}\right\rangle$. Then Eq. (19) can be expressed as:

$$
G_{M}(s)=G_{N}^{o} \Gamma \mathcal{K}^{\top}-\left\langle M^{\beta s}\right\rangle
$$

Similarly, the Mellin transform of the relaxation modulus (Eq. 15) when $0<s<$ 1 is:

$$
\begin{aligned}
& G_{M} \equiv \frac{2}{\pi} \int_{0}^{\infty}\left(\int_{0}^{\infty} t^{s-1} \sin t \underline{d} t\right) \frac{G^{\prime}}{\omega} d \omega=\frac{2}{\pi} \Gamma \sin \left(\frac{\pi s}{2}\right) \int_{0}^{\infty} \frac{G^{\prime}}{\omega^{s+1}} d \omega \text { (21a) } \\
& G_{M} \equiv \frac{2}{\pi} \int_{0}^{\infty}\left(\int_{0}^{\infty} t^{s-1} \cos t d t\right) \frac{G^{\prime \prime}}{\omega} d \omega=\frac{2}{\pi} \Gamma \cos \left(\frac{\pi s}{2}\right) \int_{0}^{\infty} \frac{G^{\prime \prime}}{\omega^{s+1}} d \omega(21 \mathrm{~b})
\end{aligned}
$$

Eqs. (20) and (21) can be combined to yield an expression for arbitrary moments of $M$ with respect to the distribution function $W(M)$ as a function of the experimental dynamic moduli:

$$
\left\langle M^{\beta s}\right\rangle=\frac{2 \sin \left(\frac{\pi s}{2}\right)}{\pi G_{N}^{0}{K^{\prime}}^{s}} \int_{0}^{\infty} \frac{G^{\prime}}{\omega^{s+1}} d \omega
$$




$$
\left\langle M^{\beta s}\right\rangle=\frac{2 \cos \left(\frac{\pi s}{2}\right)}{\pi G_{N}^{0} \boldsymbol{K}^{\prime s}} \int_{0}^{\infty} \frac{G^{\prime \prime} \boldsymbol{\varphi}^{-}}{\omega^{s+1}} d \omega
$$

where $0<s<1$. Taking $\beta s=n$, where $n$ is an integer, Eqs. (22a, 22b) yield the $n$th moment of the MWD. The moments and the average molecular weights of the MWD are related as follows:

$$
\begin{gathered}
\left\langle M^{-1}\right\rangle=\bar{M}_{n} \\
\left\langle M^{1}\right\rangle=\bar{M}_{w} \\
\left\langle M^{2}\right\rangle=\bar{M}_{w} \cdot \bar{M}_{z}
\end{gathered}
$$

and so forth.

It is known that $\beta>0$, so the -1 st moment of the MWD, i.e. $\bar{M}_{n}$, cannot be determined, since $s$ would be negative. However, if $\beta>2$ the +1 st and +2 nd moments of the MWD can be determined, since in these cases $s$ lies between 0 and 1. Therefore, we can use Eq. (22) to get $\bar{M}_{w}$ and $\bar{M}_{z}$ if we know $K^{\prime}, G_{N}^{0}$ and the dynamic moduli. Usually, $\mathrm{K}^{\prime}$ is an unknown parameter. However, if we focus on the polydispersity index $\overline{\mathrm{M}}_{\mathrm{z}} / \overline{\mathrm{M}}_{\mathrm{w}}=\left\langle M^{2}\right\rangle /\left\langle M^{1}\right\rangle^{2}$ the parameter $\mathrm{K}^{\prime}$ is not needed, and:

$$
\begin{aligned}
& \frac{M_{z}}{M_{w}}=G_{N}^{o} \frac{\pi}{2} \frac{\sin \left(\frac{\pi}{\beta}\right)}{\left(\sin \left(\frac{\pi}{2 \beta}\right)\right)^{2}} \frac{\int_{0}^{\infty} \frac{G^{\prime}}{\omega^{\left(2 / \beta^{+1}\right)}} d \omega}{\left(\int_{0}^{\infty} \frac{G^{\prime}}{\left.\omega^{\left(1 / \beta^{+1}\right.}\right)} d \omega\right)^{2}} \\
& \frac{M_{z}}{M_{w}}=G_{N}^{o} \frac{\pi}{2} \frac{\cos \left(\frac{\pi}{\beta}\right)}{\left(\cos \left(\frac{\pi}{2 \beta}\right)\right)^{2}} \frac{\int_{0}^{\infty} \frac{G^{\prime \prime}}{\omega^{\left(2 / \beta^{+1}\right)}} d \omega}{\left(\int_{0}^{\infty} \frac{G^{\prime \prime} \omega^{-}}{\omega^{\left(1 / \beta^{+1}\right)}} d \omega\right)^{2}}
\end{aligned}
$$


The parameter $\beta$ must fall in the range $2<\beta<\alpha$. The lower limit corresponds to the minimum $\beta$ value from which the polydispersity index $\bar{M}_{z} / \bar{M}_{w}$ can be calculated. The upper limit is determined by Eq. (13a) with the condition $\alpha-\beta>$ 0 , because any relaxation time, $\tau_{\mathrm{i}}$, of a chain of molecular weight $\mathrm{M}_{\mathrm{i}}$, must increase when $\bar{M}>M_{i}$ and diminish when $\bar{M}<M_{i}$ with respect to the relaxation time in the monodisperse system $\left(\overline{\mathrm{M}}=\mathrm{M}_{\mathrm{i}}\right)$.

Therefore, only the master curves of $\mathrm{G}^{\prime}(\omega)$ and $\mathrm{G}^{\prime \prime}(\omega)$ are required to calculate $\overline{\mathrm{M}}_{z} / \overline{\mathrm{M}}_{\mathrm{w}}$ since the plateau modulus is also calculated from Eq. (15b) by applying the time limit to the relaxation modulus $\left(\lim G(t)_{t \rightarrow 0}=G_{N}^{0}\right)$ :

$$
G_{N}^{0}=\frac{2}{\pi} \int \frac{G^{\prime \prime}}{\omega} d \omega
$$

It should be emphasised that the entire master curves, extending from the terminal zone to the rubbery plateau region, are needed to calculate the complete integrals. Otherwise, reliable values for the polydispersity index will not be obtained.

We have analyzed the effect of the experimental broadening of the spectrum, for monodisperse polymer, on the polydispersity index calculated, in polydisperse systems, with Eq. (24). As we mentioned above, the spectrum for a simple exponential function, in the model relaxation function, is narrower than the experimental spectrum for monodisperse polymers. Eq. (24) calculates the polydispersity index from a relaxation time distribution (RTD). If a monodisperse system has a polydisperse RTD, the calculated polydispersity index will be greater than 1. Therefore, when we calculate, with Eq. (24), the polydispersity index, in polydisperse systems, we obtain a polydispersity index, which is the 
product of two numbers: one that is the true molecular polydispersity index and the other that is related with the RTD of the monodisperse chains. Then, if we apply Eq. (24) to experimental monodisperse system data, the polydispersity index obtained will be a factor that can be used to calculate the true molecular polydispersity index of polydisperse systems. The true molecular polydispersity index of polydisperse systems can be obtained by dividing their calculated polydispersity index by this factor. This correction, due to the non-monodipersity of the RTD in monodisperse systems, seems to be unimportant in the calculation of molecular polydispersity indexes, as we can see from the comparison between experimental and calculated molecular polydispersity indexes obtained with Eq. (24).

As mentioned above, the steady state compliance is strongly dependent on polydispersity and, according to our theoretical approach, this dependence must be obtained as follows.

From the proposed relaxation modulus (Eq. 14), the relaxation spectrum, $H(\tau)$, can be related to the MWD by [8]

$$
H \longleftarrow \frac{G_{N}^{o}}{\beta} M W(M)
$$

where the relaxation time, $\tau$, is described by Eq. (13b). In addition, the steady state compliance can be calculated from the relaxation spectrum by [38]

$$
J_{e}^{0}=\frac{\int_{0}^{\infty} \tau H \leftarrow d \tau}{\left(\int_{0}^{\infty} H \smile d \tau\right)^{2}}
$$

Combining equations (13b), (25) and (26), and taking $d \tau=\beta K^{\prime} M^{\beta-1} d M$, the following $\mathrm{J}_{\mathrm{e}}^{0}$ dependence on MWD is obtained 


$$
J_{e}^{0}=\frac{1}{G_{N}^{o}} \frac{\left\langle M^{2 \beta}\right\rangle}{\left\langle M^{\beta}\right\rangle^{2}}
$$

Note that Eq. (28) is a general relationship, for which any value of $\beta$ is possible, provided that it lies in the correct range of $2-\alpha$. Therefore, the $\mathrm{J}_{\mathrm{e}}^{0}$ molecular dependence is not restricted to integer moments of $\mathrm{W}(\mathrm{M})$. In particular, it can be observed that Eq. (28) includes the relationships predicted by Doi-Edwards (Eq. 10) and Bird et al. (Eq. 11), which can be formulated as a special case for $\beta=3$ and $\beta=2$, respectively.

For the particular case of a logarithmic-normal type distribution, the model predicts that $J_{\mathrm{e}}^{0}$ scales with the polydispersity index $\overline{\mathrm{M}}_{\mathrm{z}} / \overline{\mathrm{M}}_{\mathrm{w}}$ according to

$$
J_{e}^{0} \propto\left(\frac{\bar{M}_{z}}{\bar{M}_{w}}\right)^{\beta^{2}} \quad \text { for } 2<\beta<\alpha \approx 3.4
$$

Given that $\beta$ must be greater than 2 , the power exponent with which $\mathrm{J}_{\mathrm{e}}^{0}$ scales with $I_{p 2}=\bar{M}_{z} / \bar{M}_{w}$ will be greater than 4. This leads to greater differences with the empirical value of 3.7 [18] as $\beta$ goes up. However, Agarwal [19] examined several sets of data from the literature and concluded that while the equation he proposed (Eq. 3) holds for all of them, Eq. (2) does not. Indeed, scaling exponents higher than 5.3 have been reported.

It is worth noting that the MWD dependence of $\mathrm{J}_{\mathrm{e}}^{0}$ predicted by the model (Eq. 28) is the same as that predicted by a generalized form of Ninomiya's blending law (Eq. 5). This generalized blending law is based on the following equations:

$$
H(\tau)=\sum_{i} w_{i} \cdot H_{i} \leftarrow / \lambda_{i}
$$


where $\lambda_{i}=\bar{M} / M_{i}{ }^{\lambda}$ and $\delta=\alpha-\beta$,

$$
\begin{aligned}
& \eta_{i}=\int_{0}^{\infty} H_{i} \lessdot \mathcal{d} \tau \propto M_{i}^{\alpha} \\
& J_{e i}=\frac{1}{\eta_{i}{ }^{2}} \int_{0}^{\infty} \tau H_{i} \lessdot d \tau \propto\left(M_{i}^{\alpha}\right)
\end{aligned}
$$

\section{Experimental}

\subsection{Polymer samples}

Different kinds of polymers with different degrees of polydispersity were analysed. They were: polydimethylsiloxane (PDMS), polyisoprene (PI), two series of polystyrene (PS), polydisperse and nearly monodisperse PS, and a random co-polymer of ethylene and propylene (EP). Binary blends of PDMS, PI and nearly monodisperse PS were also studied. Some of the samples analysed in this study had also been analysed in the previous work [8]. However, binary blends and nearly monodisperse PS are new. Moreover, the previous study focused on unimodal log-normal MWD while the present study extends to the polydispersity index for any kind of MWD, be it unimodal or bimodal.

The rheology of PDMS and PI samples was analysed in our laboratory. They were purchased from Aldrich, who also supplied the average molecular weight information. Three bimodal PDMS mixtures were prepared by blending a sample of low molecular weight (PDMS_L) with another of high molecular weight (PDMS_H), adding either $75 \%, 46 \%$ or $25 \%$ (in weight) of the latter. In what follows, the letters $\mathrm{H}$ and $\mathrm{L}$ in the short forms stand for high and low molecular weight component respectively. Two binary PI blends were also 
prepared in the same way, with $90 \%$ and $80 \%$ of the high molecular weight component (PI_H).

The molecular weight distributions for the three polymers examined (PDMS_H, PDMS_L and PI_H) were determined by GPC in a Waters liquid chromatograph with a light scattering detector at $75^{\circ} \mathrm{C}$. The polymer solutions were prepared at a concentration of $0.05 \%$ in toluene and injected into the system at an injection volume of $50 \mu \mathrm{L}$. A Styragel HR 5E column was used with toluene as the mobile phase at a flow rate of $0.35 \mathrm{~mL} / \mathrm{min}$. The average molecular weights of the blends were calculated from the known composition of the mixture [41].

Rheological data for EP and the polydisperse PS samples were taken, with permission, from the work of Wasserman and Graessley [42]. The PS sample was prepared by mixing nearly monodisperse polystyrenes in order to get a precise logarithmic-normal distribution (referred to as sample M2 in the work of Wasserman and Graessley [42]).

Other PS rheological data are also reported in the present study, and these were generously provided by Maier et al. [43]. They consist of a narrowly distributed PS sample (PS_L), referred to as PS60 in the work of Maier et al. [43], and a series of bimodal mixtures of this polymer, made by solution blending, with $10 \%, 20 \%$ and $40 \%$ (in weight) of a high molecular weight PS (PS_H) (referred to as PS177 in the work of Maier et al. [43]). As before, the average molecular weights of the blends were calculated from the known composition of the mixture [41].

The binary blends of the polymers analysed in the present study are hereinafter referred to by their short form: POLYMER_HOOLO0, where 00 
indicates the content (\% in weight) of high $(\mathrm{H})$ and low $(\mathrm{L})$ molecular weight components.

The average molecular weights and the polydispersity indices $I_{p 1}$ and $I_{p 2}$ of all the samples investigated are listed in Table 1.

\subsection{Rheological measurements}

The rheological data of the samples analysed in our laboratory were obtained by dynamic oscillatory tests in a controlled stress rheometer (HAAKE RS100) with parallel plates of $20 \mathrm{~mm}$ diameter $(\mathrm{gap}=0.4 \mathrm{~mm}$ ), except for the PDMS_L sample for which a cone-and-plate sensor of $20 \mathrm{~mm}$ diameter and $2^{\circ}$ angle was used. All measurements were taken in the linear viscoelastic region. The PDMS samples were analysed at three different temperatures: $50 \stackrel{\circ}{\circ}, 0 \stackrel{\circ}{\mathrm{C}}$ and $-40{ }^{\circ} \mathrm{C}$ $\left(-50 \stackrel{\circ}{ } \mathrm{C}\right.$ for the PDMS_L sample). Those of PI were analysed at $80{ }^{\circ} \mathrm{C}$ and 110 ${ }^{\circ} \mathrm{C}$. All PI samples were studied under nitrogen atmosphere in order to prevent oxidative degradation. The isotherms were shifted in order to build up the master curves at a reference temperature of $\mathrm{T}_{0}=0 \stackrel{\circ}{\mathrm{C}}$ and $110^{\circ} \mathrm{C}$ for PDMS and PI, respectively, using the time-temperature superposition principle.

Details of the rheological characterisation of the other polymers used in this study can be found in the original papers [42, 43].

Figures 1 to 3 show the dynamic moduli G' and G" master curves for the PDMS, PI and PS mixtures series. Figures 4 and 5 show respectively the dynamic modulus master curve data for EP and polydisperse PS samples.

\section{Results and discussion}


The aim of the proposed method was to calculate the polydispersity index, $\mathrm{I}_{\mathrm{p} 2}$, from the dynamic moduli of linear polymers. This was done using equations (24a or b) and the plateau modulus $\left(\mathrm{G}_{\mathrm{N}}^{0}\right)$ obtained through Eq. (25). To calculate both parameters, $I_{\mathrm{p} 2}$ and $\mathrm{G}_{\mathrm{N}}^{0}$, several integrals must be calculated from zero to infinity with respect to frequency. Therefore, experimental frequency window must be large enough to calculate these integrals accurately.

\subsection{Frequency window requirements}

The whole frequency evolution of the dynamic moduli, G' and G", from the terminal to the rubbery plateau region, is needed to achieve accurate polydispersity indices. This means that G' must evolve from a two scaling law

with $\omega\left(G^{\prime} \sim \omega^{2}\right)$ at the low frequency range (terminal zone) to a constant value of $G^{\prime}$, namely $G_{N}^{0}$, at the high frequency range (plateau region). In addition, $G^{\prime \prime}$ must evolve from a scaling law behaviour with a power exponent equal to one (G" $\sim \omega)$ to a scaling behaviour with a power exponent characteristic of the polymeric system $\left(G^{\prime \prime} \sim \omega^{-n}\right)$. It should be noted that quality dynamic data in the terminal region are more readily obtained by observing the scatter of the storage dynamic shear compliance data points, $J^{\prime}=G^{\prime \prime} /\left(G^{\prime 2}+G^{\prime \prime 2}\right)$, as $J_{e}^{0}$ is asymptotically approached. In the terminal zone only functions not influenced by viscous deformation or relaxation help ascertain the level of precision of dynamic properties [44]. On the other hand, since polymers with narrow MWD have $\mathrm{J}_{\mathrm{e}}^{0 \text { 's }}$ which are 2.5 to 3.0 times larger than their entanglement plateau compliances, we have to take care that the false plateau in G' which reflects the reciprocal of $\mathrm{J}_{\mathrm{e}}^{0}$ was not interpreted as the genuine rubbery entanglement 
plateau, $G_{N}^{o}$, [44]. In these cases, we need to explore higher frequencies than those leading to the plateau where $\mathrm{G}^{\prime}$ reflects the reciprocal of $\mathrm{J}_{\mathrm{e}}^{0}$ until $G_{N}^{o}$ is finally reached. Therefore, the experimental data must extend over a wide frequency range and this can be achieved for most polymeric materials with the aid of the time-temperature superposition principle. Figures 1 to 5 show that the whole evolution from terminal to plateau region is reached in all cases. However, in some cases it is possible to get practical results by extrapolating the experimental data in both frequency limits (low and high), according to the rules given above. An example of the resulting dynamic moduli after the frequency window extension by extrapolation is shown in Figure 4.

The polydispersity index can be calculated independently from storage or loss data. Obviously, however, both sets of data must reach the same $I_{p 2}$ parameter, and this was successfully tested for several of the analysed systems. However, from a practical point of view, it is simpler to use the storage modulus $G^{\prime}(\omega)$ (Eq. 24a), since the extension of this parameter at high frequencies is easier $\left(\left|G^{\prime}(\omega)\right|_{\omega \rightarrow \infty}=G_{N}^{0}\right)$. At the same time, this material parameter can be found in the literature for most common polymers at different temperatures. Given this, we recommend using the $G$ ' modulus for the $I_{p 2}$ determination, focusing attention on the integrals being completed.

It should be pointed out, however, that the molecular model used is only applicable from the terminal to the rubbery plateau region [8]. Therefore, glassy modes, that could appear at high frequencies in the dynamic moduli master curve data, must be removed.

\subsection{Choice of the optimal parameter $\beta$}


The polydispersity index was calculated by fitting the model described by Eq. (24) to the measured dynamic data, leaving $\beta$ as an adjustable parameter. In all samples it is adjusted into the valid range of $2<\beta<3.4$ by trial and error in order to give the known experimental value of the parameter $I_{\mathrm{p} 2}$. The $I_{\mathrm{p} 2}$ parameter values were determined by SEC analysis for pure components and by calculations from the known composition of the mixture [41] for the polymer blends. These values are listed in Table II, together with parameter $\beta$ adjusted for all the analysed samples in order to have the correct $I p_{2}$ for the rheological method presented in this study.

To choose the optimal $\beta$ value valid for all the polymers examined, the evolution of the relative error in $I_{p 2}$ with $\beta$ was analysed. Figure 6 shows the results for the five different polymer series. It should be noted that the curves corresponding to the PDMS, PI and nearly monodisperse PS series show weighted mean values of the individual relative errors. The optimal $\beta$ values for these five series are: $\beta_{1}=2.5$ (for PDMS), $\beta_{2}=2.7$ (for PI), $\beta_{3}=2.5$ (for EP), $\beta_{4}$ $=2.3$ (for polydisperse PS) and $\beta_{5}=2.5$ (for nearly monodisperse PS), also listed in Table 2. The weighted mean value of $\beta$ for all the polymer series is $\beta=2.50 \pm 0.14$

which is indicated as a vertical dashed line in Fig. 6. Therefore, 2.50 was chosen as the best $\beta$ value for calculating $I_{p 2}=\bar{M}_{z} / \bar{M}_{w}$ with the proposed rheological method.

The polydispersity indices were then recalculated with $\beta=2.5$ and the obtained values are listed in Table III. The greatest discrepancies are observed for high polydisperse samples. However, except for these systems the relative 
error never exceeds $12 \%$, regardless of the polydispersity or distribution type form (unimodal or bimodal). The small discrepancies could be reduced if different $\beta$ values were assigned to the different polymer series, although this seems unnecessary from a practical point of view. All complex relaxation modulus data were established with standard experimental error. Each experimental datum was determined by conventional replication until error does not exceed $5 \%$. Special emphasis was laid on the use of the complete frequency evolution of the dynamic moduli, taking into consideration the factors pointed out in the terminal and the rubbery plateau regions.

\section{Conclusions}

This article has described a theoretical model for determining the polydispersity index, $\bar{M}_{z} / \bar{M}_{w}$, of linear polymer melts directly from experimental rheological dynamic data. The model has been shown to be useful for predicting the polydispersity of different kinds of polymer samples and has certain advantages over other rheological techniques used for this purpose. As it is based on a molecular theory, rather than empirical rules, and predicts polydispersity in an absolute mode, no calibration is needed. Furthermore, it can be applied to any entangled linear polymeric material, whatever its nature and distribution form type (uni- or bimodal). A MWD form does not have to be assumed a priori; only the storage G' and loss G" moduli need to be known. However, for an accurate calculation of the polydispersity index, the experimental dynamic data must extend across the frequency window from the terminal to the rubbery plateau region. 
The model was successfully tested on several polymers, using both data from our laboratory and experimental data already reported in the literature. This confirms the model's validity and suggests it can be applied more widely, in particular to monodisperse samples.

The model uses the parameter $\beta$, which determines the contribution of the molecular weight of a chain in its relaxation time. $\beta=2.50$ was found to be adequate for the different polymer series analysed. Only minor discrepancies were found for polydispersity indices higher than 3 .

\section{Acknowledgements}

The authors are grateful to the Spanish "Comisión de Investigación Científica y Técnica" (projects QU1999-0533, PPQ2001-0671) for the funding that enabled this work to be carried out.

\section{References}

1. Wu S. Polym Eng Sci 1985;25:122-128.

2. Tuminello WH. Polym Eng Sci 1986;26:1339-1347.

3. Mead DW. J Rheol 1994;38:1797-1827.

4. Wasserman SH. J Rheol 1995;39:601-625.

5. Carrot C, Guillet J. J Rheol 1997;41:1203-1220.

6. Maier D, Eckstein A, Friedrich Cr, Honerkamp J. J Rheol 1998;42:1153-1173.

7. Léonardi F, Majesté JC, Allal A, Marin G. J Rheol 2000;44:675-692 and Léonardi F, Allal A, Marin G. J Rheol 2002;46:209-224.

8. Llorens J, Rudé E, Marcos RM. J Polym Sci Part B: Polym Phys 2000:38:1539-1546. 
9. Thimm W, Friedrich Cr, Marth M. J Rheol 2000;44:429-438.

10. Nobile MR, Cocchini F. Rheol Acta 2001;40:111-119.

11. Ferry JD. Viscoelastic Properties of Polymers, 3rd ed. New York: Wiley, 1980. p.19.

12. Graessley WW. J Polym Sci: Polym Phys Ed 1980;18:27-34.

13. Ferry JD. Viscoelastic Properties of Polymers, 3rd ed. New York: Wiley, 1980 (Chapter 13).

14. Montfort JP, Marin G, Monge Ph. Macromolecules 1984;17:1551-1560.

15. Montfort JP, Marin G, Monge Ph. Macromolecules 1986;19:1979-1988.

16. Carrot C, Revenu P, Guillet J. J Appl Polym Sci 1996;61:1887-1897.

17. Mieras HJMA, van Rijn CFH. Nature 1968;218:865-866.

18. Mills NJ. Nature 1968;219:1249-1250.

19. Agarwal PK. Macromolecules 1979;12:342-344.

20. Kurata M. Macromolecules 1984;17:895-898.

21. Ninomiya K. J Colloid Sci 1959;14:49-58 and 1962;17:759-767.

22. Bogue DC, Masuda T, Einaga T, Onogi S. Polym J 1970;1:563-572.

23. Kurata M, Osaki K, Einaga Y, Sugie T. J Polym Sci: Polym Phys Ed 1974;12:849-869.

24. Doi M, Edwards SF. J Chem Soc. Faraday Trans II 1978;74:1789-1801; 74:1802-1817; 74:1818-1832.

25. Doi M, Edwards SF. The Theory of Polymer Dynamics. Oxford: Clarendon Press, 1986.

26. Bird RB, Curtis CF, Armstrong RC, Hassager O. Dynamics of Polymeric Liquids, Vol. 2, 2nd ed. New York: Wiley-Interscience, 1987 (Chapter 19). 27. Couarraze G, Grossiord JL, Puisieux F. Rheol Acta 1986;25:494-500. 
28. Grossiord JL, Couarraze G. Eur Polym J 1988; 24:259-264.

29. Tzoganakis C. Can J Chem Eng 1994;72:749-754.

30. Hatzikiriakos SG. Polym Eng Sci 2000;40:2279-2287.

31. Graessley WW. Adv Polym Sci 1982;47:67-117.

32. Klein J. Macromolecules 1978;11:852-858.

33. Daoud M, de Gennes PG. J Polym Sci, Polym Phys Ed 1979;17:1971-1981.

34. Tsenoglou C. ACS Polym Prep 1987;28:185-186.

35. des Cloizeaux J. Macromolecules 1990;23:4678-4687.

36. Schroeder MJ, Roland CM. Macromolecules 1999;32:2000-2003.

37. Adachi K, Wada T, Kawamoto T, Kotaka T. Macromolecules 1995;28:35883596.

38. Ferry JD. Viscoelastic Properties of Polymers, 3rd ed. New York: Wiley, 1980 (Chapter 3).

39. Sneddon IN. The Use of Integral Transforms, New York: McGraw-Hill, 1972. p.262-297.

40. Bracewell RN. The Fourier Transform. and Its Applications, 3rd ed. Boston: McGraw-Hill, 2000. p. 343-347.

41. Comstock KJ. ACS Symposium Series 1982;197:45-64.

42. Wasserman SH, Graessley WW. J Rheol 1992;36:543-572.

43. Maier D, Eckstein A, Friedrich Cr, Honerkamp J. J Rheol 1998;42:11531173.

44. Plazek, D.J. J Rheol 1992;36:1671-1690. 


\section{Tables}

Table 1. Average molecular weights (in $\mathrm{g} \cdot \mathrm{mol}^{-1}$ ) and polydispersity indices for the polymers investigated.

\begin{tabular}{|c|c|c|c|c|c|}
\hline Sample & $\overline{\mathrm{M}}_{n} \cdot 10^{-3}$ & $\overline{\mathrm{M}}_{\mathrm{w}} \cdot 10^{-3}$ & $\overline{\mathrm{M}}_{\mathrm{z}} \cdot 10^{-3}$ & $I_{p 1}^{a}$ & $\mathrm{I}_{\mathrm{p} 2}{ }^{\mathrm{a}}$ \\
\hline PDMS_H & 380 & 630 & 890 & 1.7 & 1.4 \\
\hline PDMS_H75L25 & 159 & 496 & 1027 & 3.1 & 2.1 \\
\hline PDMS_H46L54 & 96 & 341 & 934 & 3.6 & 2.7 \\
\hline PDMS_H25L75 & 74 & 228 & 786 & 3.1 & 3.5 \\
\hline PDMS_L & 59 & 94 & 140 & 1.6 & 1.5 \\
\hline PI_H & 180 & 800 & 4800 & 4.4 & 6.0 \\
\hline PI_H90L10 & 76 & 724 & 3533 & 9.5 & 4.9 \\
\hline PI_H80L20 & 48.5 & 648 & 3510 & 13.4 & 5.4 \\
\hline PI_L ${ }^{b}$ & 12.3 & 40 & 130 & 3.3 & 3.3 \\
\hline EP & 175 & 350 & 700 & 2.0 & 2.0 \\
\hline PS & 155 & 398 & 1040 & 2.6 & 2.6 \\
\hline PS_H $H^{b}$ & 172 & 177 & 182 & 1.03 & 1.03 \\
\hline PS_H40L60 & 79 & 107 & 142 & 1.35 & 1.33 \\
\hline PS_H20L80 & 67 & 84 & 113 & 1.25 & 1.35 \\
\hline PS_H10L90 & 62 & 72 & 92 & 1.16 & 1.28 \\
\hline PS_L & 58 & 60.4 & 63 & 1.04 & 1.04 \\
\hline \multicolumn{6}{|c|}{${ }^{\mathrm{a}} \mathrm{I}_{\mathrm{p} 1}=\overline{\mathrm{M}}_{\mathrm{w}} / \overline{\mathrm{M}}_{\mathrm{n}}$ and $\mathrm{I}_{\mathrm{p} 2}=\overline{\mathrm{M}}_{\mathrm{z}} / \overline{\mathrm{M}}_{\mathrm{w}}$} \\
\hline
\end{tabular}


Table 2. Values of parameter $\beta$, for all the experimented polymer samples, at which there was a fit between polydispersity indices calculated with the proposed rheological method and experimentally-derived ones.

\begin{tabular}{|c|c|c|c|}
\hline Sample & Measured $\mathrm{I}_{\mathrm{p} 2}{ }^{\mathrm{a}}$ & $\beta_{1 j}^{b}$ & $\beta_{i}^{c}$ \\
\hline PDMS_H & 1.4 & 2.7 & \\
\hline PDMS_H75L25 & 2.1 & 2.5 & \\
\hline PDMS_H46L54 & 2.7 & 2.6 & 2.5 \\
\hline PDMS_H25L75 & 3.5 & 2.1 & \\
\hline PDMS_L & 1.5 & 3.1 & \\
\hline PI_H & 6.0 & 2.7 & \\
\hline PI_H90L10 & 4.9 & 2.7 & 2.7 \\
\hline PI_H80L20 & 5.4 & 2.7 & \\
\hline EP & 2.0 & 2.5 & 2.5 \\
\hline PS & 2.6 & 2.3 & 2.3 \\
\hline PS_H40L60 & 1.33 & 2.7 & \\
\hline PS_H20L80 & 1.35 & 2.3 & 25 \\
\hline PS_H10L90 & 1.28 & 2.1 & \\
\hline PS_L & 1.04 & 2.8 & \\
\hline \multicolumn{4}{|l|}{${ }^{\mathrm{a}} \mathrm{I}_{\mathrm{p} 2}=\overline{\mathrm{M}}_{\mathrm{z}} / \overline{\mathrm{M}}_{\mathrm{w}}$} \\
\hline
\end{tabular}


Table 3. Measured and calculated polydispersity indices for all the experimental polymer samples. The calculated polydispersity indices were obtained by taking the optimal value of 2.5 for the $\beta$ parameter.

\begin{tabular}{|c|c|c|}
\hline Sample & $\begin{array}{l}\text { Measured } \\
\mathrm{I}_{\mathrm{p} 2}{ }^{\mathrm{a}}\end{array}$ & $\begin{array}{l}\text { Calculated } \\
\mathrm{I}_{\mathrm{p} 2}{ }^{\mathrm{a}}\end{array}$ \\
\hline PDMS_H & 1.4 & 1.5 \\
\hline PDMS_H75L25 & 2.1 & 2.0 \\
\hline PDMS_H46L54 & 2.7 & 2.8 \\
\hline PDMS_H25L75 & 3.5 & 2.4 \\
\hline PDMS_L & 1.5 & 1.6 \\
\hline PI_H & 6.0 & 7.9 \\
\hline PI_H90L10 & 4.9 & 6.9 \\
\hline PI_H80L20 & 5.4 & 7.0 \\
\hline EP & 2.0 & 2.0 \\
\hline PS & 2.6 & 2.3 \\
\hline PS_H40L60 & 1.33 & 1.39 \\
\hline PS_H20L80 & 1.35 & 1.26 \\
\hline PS_H10L90 & 1.28 & 1.17 \\
\hline PS_L & 1.04 & 1.05 \\
\hline
\end{tabular}




\section{Figure legends (or captions)}

Figure 1. Dynamic moduli G' and G" master curves at a reference temperature $T_{0}$ $=0{ }^{\circ} \mathrm{C}$ for the PDMS mixtures series with $0 \%(\square), 25 \%(*), 46 \%(+), 75 \%(\square)$ and $100 \%$ (प) of the high molecular weight polymer (PDMS_H). The dotted line shows the plateau modulus for polydimethylsiloxane.

Figure 2. Dynamic moduli G' and G" master curves at a reference temperature $T_{0}$ $=110 \stackrel{\circ}{ } \mathrm{C}$ for the PI mixtures series with $100 \%(\square), 90 \%(\square)$, and $80 \%(+)$ of the high molecular weight polymer (PI_H). The dotted line shows the plateau modulus for polyisoprene.

Figure 3. Dynamic moduli G' and G" master curves at a reference temperature $T_{0}$ $=170{ }^{\circ} \mathrm{C}$ for the PS mixtures series with $0 \%(\square), 10 \%(*), 20 \%(+)$ and $40 \%(\square)$ of the high molecular weight polymer. The dotted line shows the plateau modulus for polystyrene.

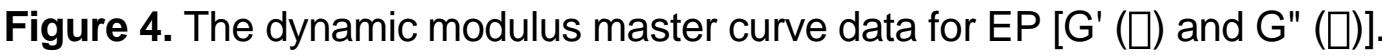

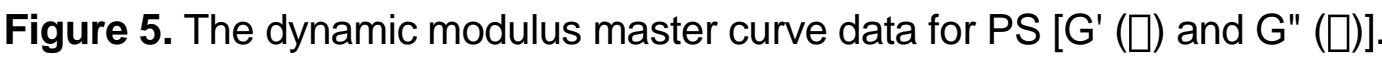

Figure 6. The relative error in $\mathrm{I}_{\mathrm{p} 2}$ with dependence on the parameter $\beta$ for the five kinds of polymers analysed: $(+)$ and full line for PDMS, $(x)$ and full line for $\mathrm{PI}$, full line for EP, dashed line for polydisperse PS and $(*)$ with full line for nearly monodisperse PS. The vertical dashed line shows the chosen optimal value of $\beta$ $(=2.5)$. 
Figure 1
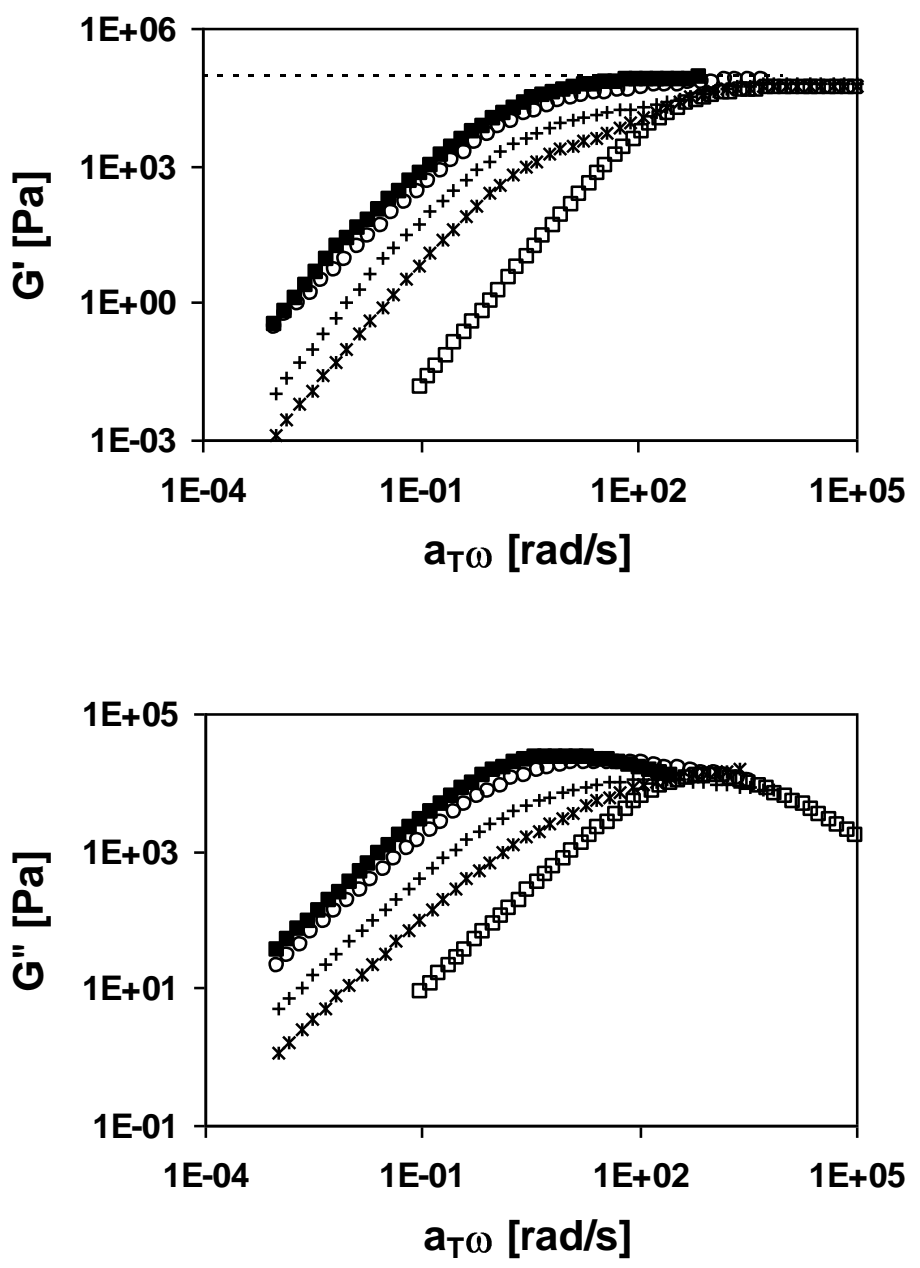
Figure 2
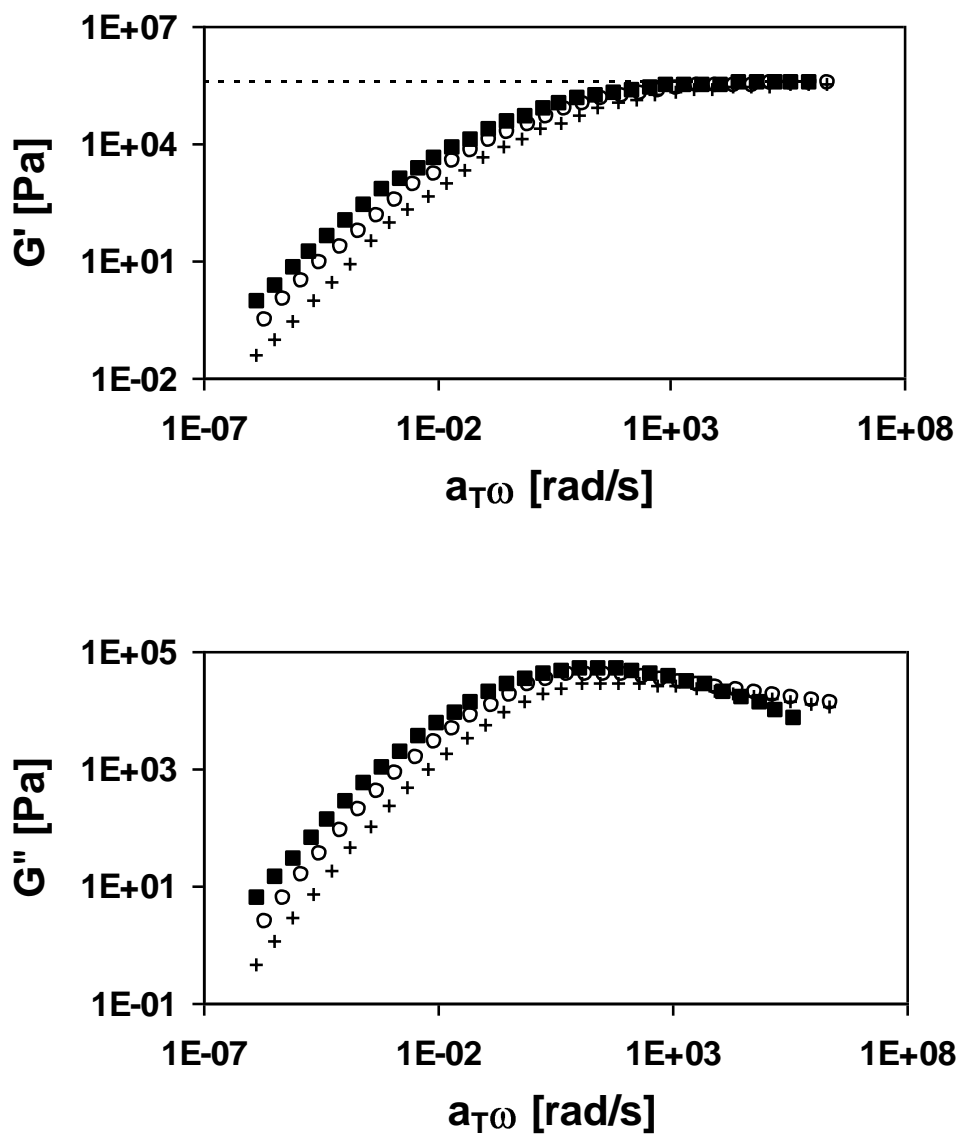
Figure 3
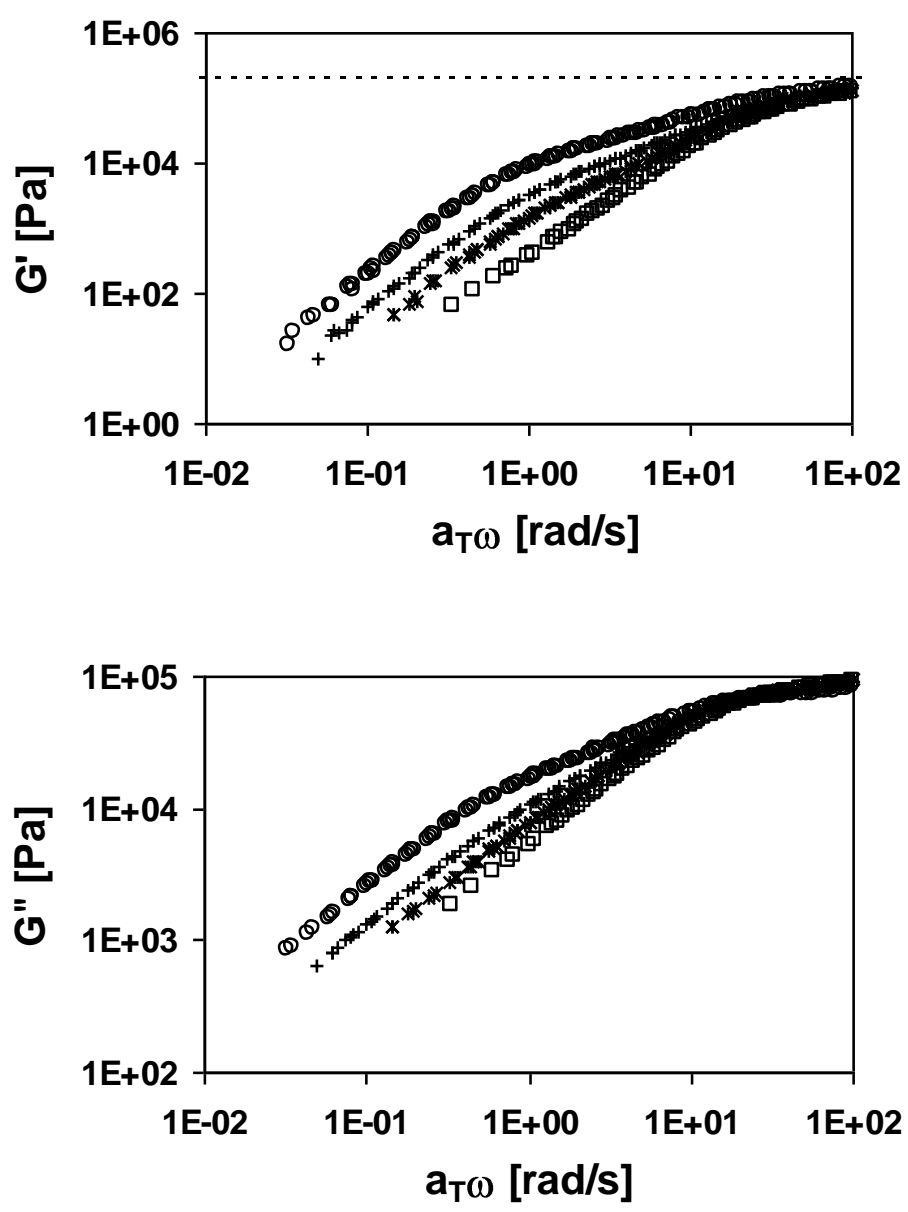
Figure 4

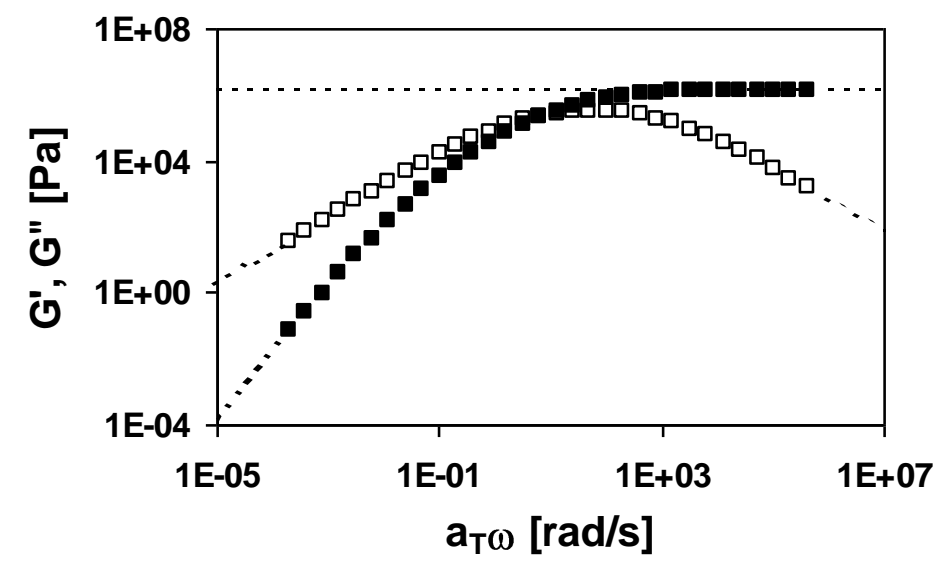


Figure 5

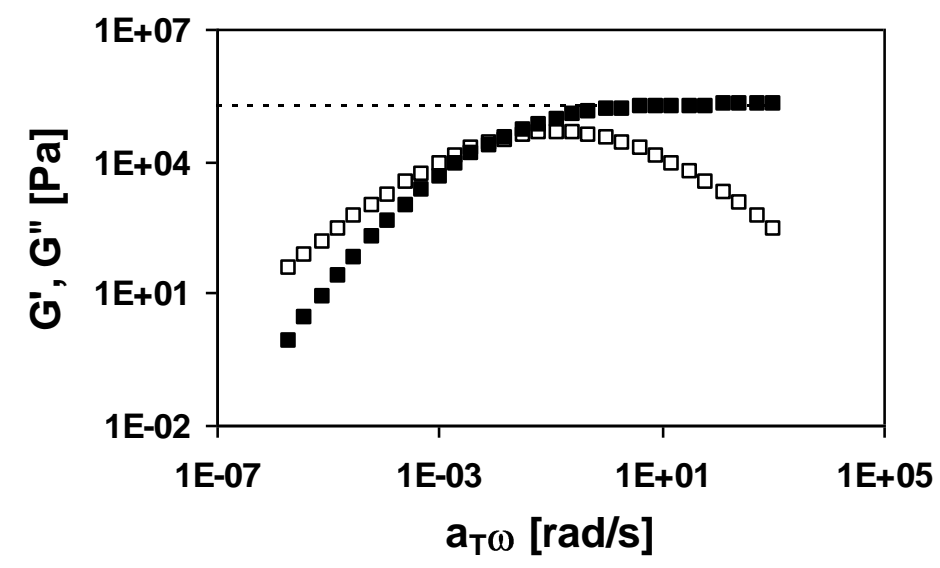


Figure 6

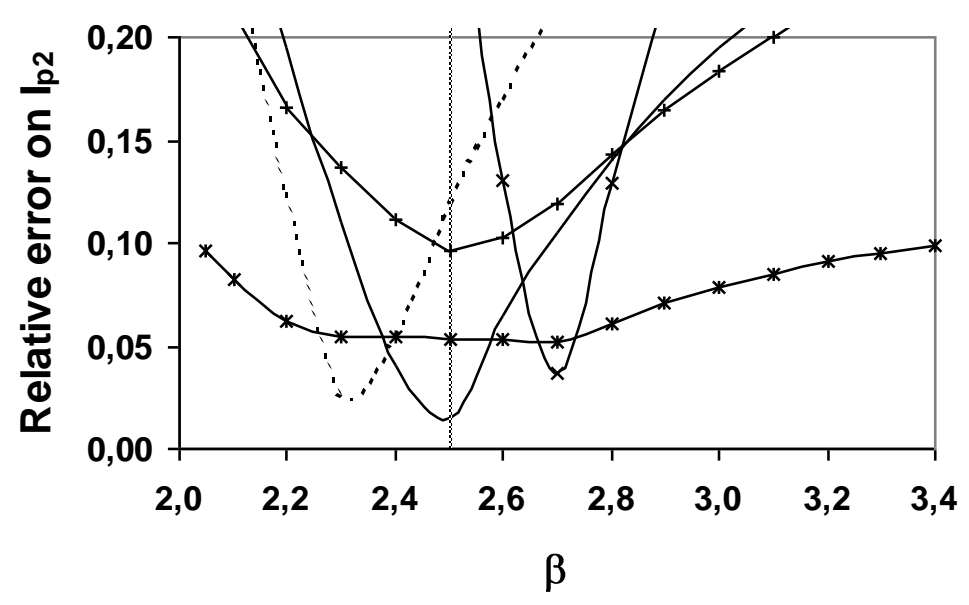

